\title{
Caractérisation de la filière porcine dans les villes de Pala et Moundou en zone des savanes du sud-ouest du Tchad
}

\author{
Youssouf Mopaté Logténé ${ }^{1 *}$ Madjina Tellah ${ }^{2}$ \\ Ali Brahim Bechir ${ }^{3}$ Alladoum Nayo ${ }^{4}$
}

\begin{abstract}
Mots-clés
Porcin, chaîne d'approvisionnement alimentaire, production animale, comportement du consommateur, Tchad
\end{abstract}

Submitted: 12 December 2017

Accepted: 20 October 2019

Published: 24 February 2020

DOI: $10.19182 /$ remvt.31291

\section{Résumé}

L'étude a caractérisé les différents segments de la filière porcine des villes de Pala et Moundou en zone des savanes au Tchad. Des enquêtes transversales et rétrospectives, associées à des observations directes, ont été menées auprès de producteurs, de transformateurs et de consommateurs répartis dans ces deux villes. Au total, 1318 porcs ont été dénombrés dont 354 femelles en âge de reproduction. Le nombre de mises bas moyen par an a été de 1,8 et la taille de la portée de 7,2 porcelets. La productivité numérique annuelle par femelle a été de 11 porcelets. Pour l'ensemble des carcasses transformées annuellement, plus de la moitié était traitée à Moundou, la seconde ville du Tchad. A Pala, les consommateurs ont préféré la viande grillée. A Moundou, les préférences ont été partagées entre la viande frite, et la viande au four ou grillée. Les résultats ont aussi montré que l'élevage de porcs visait l'amélioration des revenus de la famille grâce à la vente d'animaux, et la production d'animaux autoconsommés. Par la diversité des produits transformés, les activités de transformation et de préparation alimentaire, les acteurs de la filière ont mis à la disposition des consommateurs des aliments carnés à un coût relativement modéré. Les innovations majeures observées dans la filière étaient une porcherie améliorée, un abattoir privé, une société d'élevage et de commercialisation des viandes, et une boucherie-charcuterie pour la transformation et la distribution. La production, la transformation et la commercialisation de la viande porcine ont généré des emplois et amélioré le revenu des différents acteurs de la filière.

- Comment citer cet article : Mopaté Logténé Y., Tellah M., Bechir A.B., Nayo A., 2020. Characterization of the swine sector in the cities of Pala and Moundou in the savanna zone of Southwestern Chad. Rev. Elev. Med. Vet. Pays Trop., 73 (1): 3-10, doi: 10.19182/remvt.31291

\section{INTRODUCTION}

En Afrique au sud du Sahara, notamment dans les zones urbaines et périurbaines, les productions agricoles (animales et végétales) familiales constituent des stratégies de survie des populations pauvres (Temple et Moustier, 2004 ; Dongmo et al., 2005). Des études montrent que le maraîchage et l'élevage sont les principales activités agricoles mises en œuvre dans ces zones (Moustier et al., 2004 ; Fall et al., 2004). Elles permettent aux producteurs non seulement de disposer des protéines végétales et animales mais aussi de se procurer voire d'augmenter leurs revenus à travers la vente des surplus

1. Institut de recherche en élevage pour le développement (IRED), BP 433, Farcha, N'Djaména, Tchad.

2. Institut national supérieur des sciences et techniques d'Abéché (INSTA), Tchad.

3. Institut national supérieur d'élevage de Moussoro (INSEM), Tchad.

4. Délégation régionale de l'élevage du Sud-Ouest, Moundou, Tchad.

* Auteur pour la correspondance

Tél. : +235 66299248 ; email : mopate.ly@ gmail.com
(Mfoukou-Ntsakala et al, 2006). Ces surplus écoulés sur les marchés des villes contribuent à leur approvisionnement en denrées alimentaires (Dongmo et al., 2005). En effet, la démographie galopante des villes induit une demande urbaine sans cesse en hausse des produits alimentaires, notamment carnés, et influence considérablement les filières animales (Touré et Ouattara, 2001 ; Diao, 2004). Ainsi, l'urbanisation dynamise l'agriculture, suscite le développement d'élevages à caractère intensif, semi-intensif voire une amélioration des élevages traditionnels, et des innovations dans la production, la transformation, le stockage et la commercialisation, correspondant aux modes de vie des habitants (Guerin et Faye, 1999 ; Cirad-Emvt, 1999 ; Bricas et Seck, 2004).

En matière d'élevage dans les zones urbaines et périurbaines, pour faire face à la hausse constante de la demande et lutter contre l'insécurité alimentaire, ceux à cycles courts, notamment les porcs, s'inscrivent en bonne place. Les familles choisissent de développer l'élevage de porcs pour générer des revenus car son élevage reste important dans la diversification des ressources alimentaires et monétaires (Ossebi et al., 2018). En effet, le porc est un animal prolifique, l'investissement initial est faible et son alimentation en élevage 
traditionnel et semi-intensif repose sur des déchets et des résidus de transformation agroalimentaire (Mopaté, 2008 ; Robineau, 2018).

$\mathrm{Au}$ Tchad, la filière porcine, longtemps limitée dans son essor par un manque de débouchés (Mopaté et al., 2008), se développe aujourd'hui grâce au dynamisme des marchés camerounais et de la consommation intérieure (Koussou et Duteurtre, 2002 ; Mopaté et Koussou, 2003). Cette situation a entraîné un regain d'intérêt dans la production. Les lieux de transformation et de consommation hors foyer de viande porcine se sont ainsi multipliés dans les grandes villes du sud du Tchad et dans la capitale (Mopaté et Koussou, 2003 ; Mopaté et al., 2007a) Ces entreprises urbaines de petite taille innovent souvent en fournissant des produits transformés diversifiés et en développant des stratégies de vente susceptibles d'intéresser beaucoup de consommateurs. Si un grand nombre de travaux de recherche sur la transformation et la consommation de la viande porcine ont été réalisés dans les pays au nord (Touraille et Monin, 1984), très peu ont été conduits dans les pays au sud du Sahara, notamment au Tchad. Or, les effectifs importants commercialisés par cette filière dynamisent la production et la transformation.

Envisager une meilleure organisation de la filière, améliorer l'offre quantitative et qualitative en viande porcine, et appréhender les enjeux d'amélioration de la qualité de la viande passent par une meilleure connaissance de l'état actuel de cette activité. Aussi, les objectifs de l'étude étaient de caractériser les différents segments de la filière et de souligner les innovations apparues dans les villes de Pala et Moundou qui nécessitent d'être appuyées et encouragées.

\section{MATERIEL ET METHODES}

\section{Sites de l'étude}

Chef-lieu de la région du Logone occidental, Moundou est située à 480 kilomètres au sud-ouest de la capitale, N'Djaména, en zone agricole soudanienne. Parfois qualifiée de capitale économique du Tchad, Moundou est le siège de plusieurs unités industrielles. Sa population est constituée à $93 \%$ de chrétiens et d'animistes (Mopaté et Matna, 2012) qui consomment de la viande porcine.

Située à la même distance mais au sud de N'Djaména, Pala est le cheflieu de la région du Mayo-Kebbi Ouest, proche de la frontière avec le Cameroun. Sa population est constituée en majorité de chrétiens et d'animistes, ce qui explique là aussi l'importance de la consommation de viande de porc. La région est également grande productrice de coton et de céréales. Les deux sites d'étude sont localisés (figure 1) dans la zone des savanes au sud-ouest du Tchad.

\section{Production}

Après l'analyse de la littérature disponible, une enquête de terrain menée par les agents de l'élevage de chaque ville a permis de collecter en 2013 les données pour caractériser la filière à travers la production, la transformation et la consommation.

Pour caractériser les élevages, nous avons dû nous adapter au niveau de connaissance disponible sur les effectifs réels des producteurs de porcs sur chacun des deux sites. A Pala, l'absence d'un dénombrement préalable des éleveurs nous a amenés à exploiter les informations fournies par les groupements des producteurs. Pour ce faire, l'approche descendante des différentes organisations aux membres a été retenue afin de sélectionner les élevages, tout en élargissant l'échantillon à des exploitations voisines, non membres des groupements rencontrés. Pour sélectionner les quartiers enquêtés, la répartition spatiale des élevages dans la ville a été étudiée préalablement à l'enquête et a souligné la présence d'élevages dans les trois arrondissements de Pala. Ainsi, 46 éleveurs appartenant à 20 groupements et repartis entre les trois arrondissements ont été enquêtés. A Moundou, en revanche, l'utilisation d'un recensement préalable effectué en
2012 a permis de choisir de façon aléatoire 67 éleveurs repartis dans tous les quartiers des quatre arrondissements de la ville, de manière à obtenir un échantillon représentatif de la diversité des élevages.

Une enquête transversale et rétrospective par questionnaire a été conduite auprès de chaque producteur sur les lieux d'élevage. Les points abordés ont été les caractéristiques sociodémographiques des producteurs, les effectifs et races de porcs, la composition du troupeau (reproductrices, porcs à l'engrais et verrats), la propriété des animaux, les paramètres de reproduction, les pratiques d'élevage (habitat, alimentation et exploitation), le niveau d'organisation professionnelle, et les innovations introduites dans la pratique de l'élevage des porcs. De manière complémentaire, des observations directes ont été menées sur les porcheries, les aliments consommés au moment des repas ou ceux stockés et restés dans les mangeoires. Ces observations ont permis d'identifier les aliments les plus couramment servis aux porcs.

\section{Transformation}

Il convient de préciser que le terme transformateur inclut les boucherstransformateurs, les préparateurs et les restaurateurs. Le transformateur achète le porc, l'abat, transforme la viande et vend les produits transformés. Il est assisté par quelques personnes rémunérées à la journée. Pour caractériser ce segment, une enquête auprès des transformateurs a été conduite. En raison de leur faible effectif, la totalité de ces acteurs en activité sur les deux sites ont été enquêtés, soit six à Pala et dix à Moundou. Ces acteurs exerçaient essentiellement une activité de restauration de rue. Un seul transformateur (localisé à Moundou) était spécialisé en charcuterie porcine (jambon, saucisse, saucisson, pâté, merguez, etc.). A l'aide d'un questionnaire, des enquêtes transversales

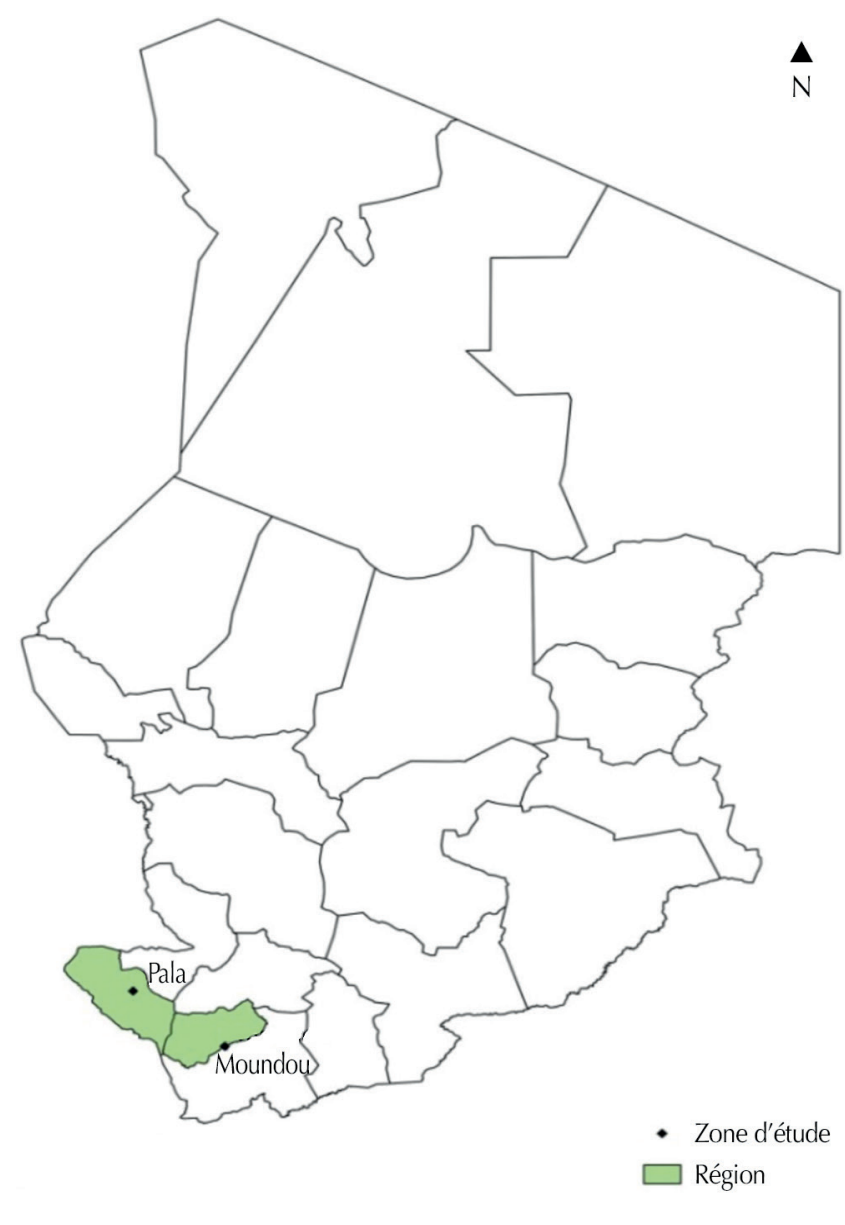

Edition : juillet 2017 Source: IRED/TRAD Réalisation : Aurélie Tobde Keilar

Figure 1 : localisation des villes de Pala et Moundou en zone des savanes au sud-ouest du Tchad. 
et rétrospectives ont été menées sur les lieux de transformation et ont porté sur le profil des acteurs, le nombre d'assistants, les dépenses et les marges bénéficiaires brutes, le niveau d'organisation professionnelle, et les innovations apportées dans la pratique de la transformation depuis le démarrage des activités. Des observations directes ont permis de caractériser les différents produits transformés. Le charcutier de Moundou a fait l'objet d'entretiens plus ouverts dans son restaurant, en utilisant un guide d'entretien. Le niveau d'organisation et les innovations introduites ont été aussi recherchés.

\section{Consommation}

Pour caractériser la consommation, une enquête transversale et rétrospective a été menée auprès de consommateurs rencontrés au hasard dans les lieux de consommation hors domicile. Ces lieux étaient le plus souvent fixes mais séparés de la partie transformation/ préparation par une simple cloison, ou implantés très près d'elle. Une pancarte avec le dessin d'un porc signalait leur présence. Le choix de consommer hors foyer a été guidé par le fait que la viande porcine était surtout consommée dans ce cadre.

Au total, 50 consommateurs à Pala et 37 à Moundou ont été enquêtés, ce qui a permis d'appréhender la diversité des pratiques alimentaires et de leurs facteurs d'évolution. Les profils et les préférences des consommateurs, les fréquences de consommation, les dépenses hebdomadaires, les raisons du choix du lieu de consommation, l'environnement du lieu, et l'ambiance générale ont été analysés. Pour chaque consommateur, les principales occasions de consommer de la viande porcine en famille ont aussi été notées. Les observations ont porté également sur les produits transformés commandés et les boissons prises au moment de la consommation. Pour les consommations en groupe, seule la personne qui payait la note a été interviewée.

\section{Analyse des données}

Les données collectées lors des enquêtes ont été saisies sous Excel et transférées dans le logiciel SPSS (SPSS, 2009) pour les analyses statistiques. Un tri à plat a été effectué pour sélectionner les variables à croiser en analyse de variance et pour rechercher des différences sur les moyennes. Les variables retenues ont été les villes et les catégories des acteurs. En ce qui concerne les données d'élevage, le taux d'exploitation a été calculé par le rapport des effectifs exploités (ventes et autoconsommation) sur les effectifs totaux recensés dans les élevages.

\section{RESULTATS}

\section{Production}

\section{Caractéristiques des producteurs}

Dans les deux villes, les éleveurs étaient relativement jeunes, mariés et scolarisés pour la plupart. Le pourcentage de producteurs instruits était de $81 \%$ à Moundou et de $63 \%$ à Pala. En moyenne ils étaient âgés de 42 ans, et leurs familles comportaient dix personnes, dont quatre à cinq étaient actives (tableau I). Globalement pour les deux villes, les hommes constituaient $64 \%$ des chefs d'atelier porcin contre $36 \%$ des femmes.

\section{Pratiques de production}

Plus de la moitié (51\%) des élevages ont été mis en place dans les années 2000, contre $34 \%$ en 1990, $11 \%$ en 1980 et seulement $4 \%$ dans les années 1960 et 1970. Concernant les pratiques de conduite, la quasi-totalité des producteurs (99\%) disposait d'un abri que les animaux occupaient le plus souvent en saison de pluies.

La majorité des producteurs de Pala (85 \%) et de Moundou (94\%) alimentaient les porcs toute l'année, avec une régularité plus observée à Pala qu’à Moundou. Les aliments les plus souvent distribués étaient les drêches artisanales et les sons de céréales, complétés de temps en temps par les déchets de cuisines familiales et, dans une moindre mesure, les graines ou tourteaux de coton.

La propriété du troupeau était surtout individuelle (77\%) dans les deux villes. La plupart des producteurs (97\%) élevaient des porcs de race locale indigène. L'enquête a porté sur 1318 porcs dont $35 \%$ étaient à Pala et $65 \%$ à Moundou. Des différences significatives sur les effectifs moyens de porcs par élevage ont été observées $(p<0,001)$ entre les deux villes. Les femelles en âge de reproduction étaient au nombre de 354 dont $33 \%$ à Pala et $67 \%$ à Moundou. La moyenne des femelles en âge de reproduction par élevage a varié entre deux et quatre femelles. La moyenne de verrats par troupeau a été moins élevée à Pala (1 verrat) qu'à Moundou (2 verrats). Au total 252 porcelets sont morts avant sevrage, répartis pour $73 \%$ à Moundou contre seulement $27 \%$ à Pala. La productivité numérique moyenne annuelle par truie était de 11 porcelets. Elle était plus élevée $(\mathrm{p}<0,01)$ à Pala qu'à Moundou. En revanche, les mortalités moyennes avant sevrage ont été plus élevées ( $\mathrm{p}<0,05)$ à Moundou qu’à Pala (tableau II).

\section{Fonctions et relations}

La production de porcs dans les deux villes générait des revenus issus de la vente, et des produits pour l'autoconsommation en vue d'améliorer le bien-être de la famille. Ces fonctions ont été largement exprimées par les différents acteurs. Les recettes de vente servaient surtout à l'achat des céréales. Certains producteurs ont pu réaliser des projets aux investissements relativement importants avec l'élevage porcin, par exemple la construction d'écoles privées, l'acquisition

\section{Tableau I}

Profil des producteurs de porcs à Pala et Moundou au sud-ouest du Tchad

\begin{tabular}{lcc} 
Profil producteur & Pala $(\mathbf{n}=\mathbf{4 6})$ & Moundou $(\mathbf{n}=\mathbf{6 7})$ \\
\hline Age moyen (années) & $41,61 \pm 12^{\mathrm{a}}$ & $42,91 \pm 12,4^{\mathrm{b}}$ \\
Homme $(\%)$ & 50 & 73 \\
Femme $(\%)$ & 50 & 27 \\
Nb. moyen de membres & $10,33 \pm 6,2^{\mathrm{a}}$ & $10,81 \pm 5,8^{\mathrm{a}}$ \\
de la famille & & \\
Nb. moyen d'actifs & $4,80 \pm 6,2^{\mathrm{a}}$ & $4,12 \pm 2,6^{\mathrm{b}}$ \\
Marié $(\%)$ & 89 & 84 \\
Analphabète $(\%)$ & 37 & 19 \\
Instruit $(\%)$ & 63 & 81
\end{tabular}

a,b Les moyennes sur une même ligne suivies de lettres différentes sont significativement différentes au seuil de $5 \%$.

\section{Tableau II}

Structure et paramètres de reproduction annuels des élevages porcins à Pala et Moundou au sud-ouest du Tchad

\begin{tabular}{lcc} 
Structure et paramètre & Pala & Moundou \\
\hline Effectif moyen & $9,9 \pm 6,7^{\mathrm{a}}$ & $12,9 \pm 10,4^{\mathrm{b}}$ \\
Nb. moyen de verrats & $1,2 \pm 0,40^{\mathrm{a}}$ & $1,7 \pm 0,82^{\mathrm{b}}$ \\
Femelles en reproduction & $2,52 \pm 1,80^{\mathrm{a}}$ & $3,55 \pm 4,20^{\mathrm{b}}$ \\
Nb. moyen de mises bas & $1,91 \pm 0,51^{\mathrm{a}}$ & $1,87 \pm 0,34^{\mathrm{a}}$ \\
Taille de la portée à la mise bas & $7,2 \pm 1,5^{\mathrm{a}}$ & $7,3 \pm 1,8^{\mathrm{a}}$ \\
Mortalités moyennes avant sevrage & $2,76 \pm 1,27^{\mathrm{a}}$ & $3,73 \pm 2,22^{\mathrm{b}}$ \\
Moyenne porcelets sevrés & $12,41 \pm 4,05^{\mathrm{a}}$ & $10,67 \pm 3,57^{\mathrm{b}}$ \\
Productivité moyenne & $11,38 \pm 3,85$
\end{tabular}

${ }^{\mathrm{a}, \mathrm{b}}$ Les moyennes sur une même ligne suivies de lettres différentes sont significativement différentes au seuil de $5 \%, 1 \%$ et $10 \%$. 
de moyens de transport comme des motocyclettes, ou l'acquisition d'équipements agricoles. Evoqué aussi par les producteurs citadins, la production porcine contribuait également à la réalisation des travaux champêtres (labour, sarclage et récolte). En effet, ces travaux exécutés par des groupes de jeunes étaient rétribués par des porcs vifs. Les producteurs étaient souvent en relation avec les préparatrices de bières et d'alcool traditionnel pour la récupération des drêches artisanales moyennant la fourniture de deux porcelets par an, ou le paiement d'argent liquide après quelques mois.

\section{Transformation}

\section{Caractéristiques des transformateurs}

Les seize transformateurs enquêtés (en dehors du charcutier) étaient des hommes âgés en moyenne de 31 ans, sans différence significative entre les deux villes ( $p>0,05)$. Chaque transformateur avait en moyenne deux assistants. L'ethnie Ngambaye était majoritaire (11/16), le reste était composé de Moundang (2), Kera (1), Laka (1) et Mouroum (1). La grande majorité (14/16) exerçait la transformation de la viande comme activité principale, les autres pour l'agriculture. Globalement, la plupart des transformateurs (75\%) avaient démarré leur activité dans les années 2000 (tous étaient de Moundou), contre $19 \%$ dans les années 1990, et $6 \%$ dans les années 1980. Sur l'ensemble, $94 \%$ avaient été scolarisés dont $56 \%$ au niveau du primaire seulement, et $38 \%$ jusqu'au secondaire. Un seul transformateur analphabète a été identifié à Moundou.

\section{Pratiques de transformation}

En un an, 1256 carcasses ont été traitées à Pala et 1821 à Moundou. La moyenne globale par transformateur était de $192 \pm 87$ carcasses par an, avec 209 carcasses à Pala et 182 à Moundou. Les porcs étaient achetés dans les deux villes respectives ou dans leur périphérie. Les moyens de transport étaient surtout le pousse-pousse à bras (56\%), la conduite à pieds $(31 \%)$ et le vélo $(13 \%)$. Tous les transformateurs privilégiaient dans leur choix les porcs sans kyste de cysticerque, en pratiquant systématiquement le test de langueyage dans les différents lieux d'achat. Les autres critères de choix des porcs étaient la conformation (44\% des transformateurs), l'embonpoint (38\%), l'état sanitaire général $(13 \%)$ et le poids $(5 \%)$. Les principaux produits transformés étaient par ordre d'importance la viande grillée, la viande frite et la soupe à la viande. A Pala, la viande et le lard grillés étaient découpés en lamelles et vendus dans les lieux de consommation. La tête était soit grillée, soit transformée en soupe pour être vendue.

\section{Fonction sociale, flux et exploitation}

La transformation de la viande porcine a contribué de manière appréciable dans les recettes journalières des acteurs. Elle a résorbé le chômage par la création d'emplois pour les 16 bouchers-transformateurs et leurs 32 assistants. Elle a contribué, avec les prix pratiqués et la diversité des produits transformés, à mettre à la disposition de la population consommatrice des protéines animales à moindre coût. La majorité des transformateurs (13/16) entretenaient des relations d'affaires (70\%), de parenté (15\%) ou d'amitié (15\%) avec les producteurs.

Concernant les 613 porcs vendus dans les deux villes entre janvier et décembre 2012, les bouchers-transformateurs ont été les principaux clients (457 têtes soit $75 \%$ ), suivis des particuliers (94 soit $15 \%$ ) et des revendeurs (62 soit $10 \%$ ). Les effectifs autoconsommés par an ont été de 199 porcs dont $66 \%$ à Moundou et $44 \%$ à Pala. Dans les lieux de transformation hors foyer, parmi les 3077 carcasses transformées annuellement, $46 \%$ ont été traitées en saison sèche et $54 \%$ en saison des pluies dont $41 \%$ à Pala et $59 \%$ à Moundou.

Le taux d'exploitation (vente et autoconsommation) a avoisiné $62 \%$. La majorité des acheteurs dans les deux villes étaient des boucherstransformateurs qui pour la plupart payaient comptant (tableau III).
Selon les villes et les catégories de clients, les variations des prix moyens par type d'acheteurs ont été plus significatives $(\mathrm{p}<0,05)$ à Pala où les revendeurs ont payé plus cher les porcs. Des variations significatives des prix par catégorie de client n'ont pas été observées à Moundou (tableau IV).

\section{Consommation hors foyer}

\section{Caractéristiques des consommateurs}

Au total, 87 consommateurs hors foyer - $57 \%$ à Pala et $43 \%$ à Moundou - étaient concernés. La moyenne d'âge était de 30 ans. Ceux de Pala étaient un peu plus âgés (31 ans) que ceux de Moundou (29 ans), sans différence significative. Les femmes étaient moins âgées que les hommes ( $\mathrm{p}<0,05)$. Pour l'ensemble des deux villes, les consommateurs étaient surtout des hommes mariés, scolarisés, fidèles à un lieu de consommation pour la qualité des produits transformés (tableau V).

\section{Pratiques de consommation}

La quasi-totalité (99\%) des produits transformés était consommée sur place. Dans les lieux de transformation et de consommation implantés pour l'essentiel autour des débits de boisson, la fréquence moyenne hebdomadaire de consommation a été significativement ( $\mathrm{p}<0,05)$ plus élevée à Moundou (cinq fois) qu’à Pala (quatre fois). Les produits transformés consommés en premier choix étaient la

\section{Tableau III}

Exploitation annuelle de la production porcine et mode de paiement à Pala et à Moundou au sud-ouest du Tchad ( $\mathrm{n}=$ nombre d'élevages)

\begin{tabular}{lcc} 
Exploitation & $\begin{array}{c}\text { Pala } \\
(\mathbf{n = 4 6 )}\end{array}$ & $\begin{array}{c}\text { Moundou } \\
(\mathbf{n = 6 2})\end{array}$ \\
\hline Autoconsommation moyenne & $2,03 \pm 1,51$ & $2,40 \pm 2,51$ \\
Vente moyenne de porcs & $6,37 \pm 6,62$ & $5,16 \pm 5,47$ \\
Prix moyen (FCFA) & $12150 \pm 4680$ & $11435 \pm 5025$ \\
Age moyen (mois) & $11,60 \pm 4,68$ & $11,92 \pm 4,99$ \\
Acheteur (boucher- & 87 & 71 \\
transformateur) (\%) & & 84 \\
Paiement comptant (\%) & 96 &
\end{tabular}

\section{Tableau IV}

Variation des effectifs, de l'âge et du prix moyen des porcs par catégorie de client à Pala et Moundou au sud-ouest du Tchad

\begin{tabular}{llccc} 
Ville & $\begin{array}{l}\text { Catégorie } \\
\text { client }\end{array}$ & Nb. porcs & Age (mois) & $\begin{array}{c}\text { Prix moyen } \\
\text { (FCFA) }\end{array}$ \\
\hline \multirow{2}{*}{ Pala } & $\begin{array}{l}\text { Transformateur } \\
\text { Tarticulier }\end{array}$ & 257 & 11,9 & $11725^{\mathrm{a}}$ \\
& $\begin{array}{l}\text { Pavendeur } \\
\text { Moundou }\end{array}$ & 14 & 12 & $20000^{\mathrm{c}}$ \\
\cline { 2 - 4 } & Transformateur & 200 & 11,6 & 10900 \\
& Particulier & 72 & 13,8 & 13360 \\
& Revendeur & 48 & 11 & 11750
\end{tabular}

* Prix moyen du porc vif selon l'âge et le type de client ; ${ }^{a, b, c}$ Les moyennes des prix par catégorie de client à Pala, suivies de lettres différentes sont significativement différentes au seuil de $5 \%$. 
viande frite et au four à Moundou, la viande grillée à Pala. Pour 23 consommateurs, les produits transformés consommés en deuxième choix étaient surtout la soupe de viande, suivie des côtelettes frites, de la tête et des oreilles (tableau VI).

Selon la profession des consommateurs, les salariés et les personnes exerçant des petits métiers avaient des fréquences de consommation

\section{Tableau V}

Profil des consommateurs hors foyer à Pala et Moundou au sud-ouest du Tchad

\begin{tabular}{|c|c|c|}
\hline Paramètre & & Valeur \\
\hline Sexe $(\%)$ & $\begin{array}{l}\text { Homme } \\
\text { Femme }\end{array}$ & $\begin{array}{c}92 \\
8\end{array}$ \\
\hline Age moyen (années) & $\begin{array}{l}\text { Homme } \\
\text { Femme }\end{array}$ & $\begin{array}{l}30,89 \pm 9,70^{a} \\
22,29 \pm 5,77^{b}\end{array}$ \\
\hline Statut (\%) & $\begin{array}{l}\text { Marié } \\
\text { Célibataire } \\
\text { Divorcé }\end{array}$ & $\begin{array}{c}56 \\
39 \\
5\end{array}$ \\
\hline Activité exercée (\%) & $\begin{array}{l}\text { Etudiant/élève } \\
\text { Petit métier } \\
\text { Salarié } \\
\text { Agriculteur }\end{array}$ & $\begin{array}{l}28 \\
26 \\
25 \\
21\end{array}$ \\
\hline Scolarisation (\%) & $\begin{array}{l}\text { Scolarisé } \\
\text { Non scolarisé }\end{array}$ & $\begin{array}{c}91 \\
9\end{array}$ \\
\hline Niveau d'instruction (\%) & $\begin{array}{l}\text { Primaire } \\
\text { Secondaire } \\
\text { Supérieur }\end{array}$ & $\begin{array}{l}32 \\
40 \\
19\end{array}$ \\
\hline $\begin{array}{l}\text { Fidélisation à un lieu de } \\
\text { consommation }(\%)\end{array}$ & $\begin{array}{l}\text { Fidèle } \\
\text { Non fidèle }\end{array}$ & $\begin{array}{l}52 \\
48\end{array}$ \\
\hline $\begin{array}{l}\text { Raison de fidélisation à un } \\
\text { lieu }(\%)\end{array}$ & $\begin{array}{l}\text { Qualité produits } \\
\text { transformés } \\
\text { Environnement / } \\
\text { cadre agréable } \\
\text { Lien de parenté } \\
\text { ou d'amitié } \\
\text { Prix pratiqué }\end{array}$ & $\begin{array}{l}71 \\
13 \\
12 \\
4\end{array}$ \\
\hline
\end{tabular}

hebdomadaires plus élevées (cinq fois) que la moyenne. En revanche, les étudiants et les élèves avaient une fréquence hebdomadaire trois fois plus faible que la moyenne à Pala et six fois plus faible à Moundou. La plupart des consommateurs ont consommé ces produits en compagnie de trois personnes en moyenne.

\section{Fonctions sociale et ventilation des prix}

La consommation de porc, comme rapporté par tous les niveaux socioprofessionnels, a amélioré la ration alimentaire quotidienne. Elle a contribué, à travers le regroupement de personnes (84\% des cas), à renforcer les liens familiaux et d'amitié. Ces liens de parenté ou d'amitié avec le transformateur étaient faibles (12\%). Selon le morceau découpé, dans les deux villes, la viande grillée a coûté 100 FCFA, la viande frite 210 FCFA, la viande au four 250 FCFA et la soupe $200 \mathrm{FCFA}$ le plat. Ces variations de prix moyen étaient significatives $(\mathrm{p}<0,01)$. La marge moyenne brute des transformateurs a été de 2580 FCFA à Pala et de 2650 FCFA à Moundou, sans différence significative. En fonction des différents produits transformés, la soupe a procuré une marge brute de 2330 FCFA, la viande grillée de 2570 FCFA et la viande frite de 2800 FCFA.

Des variations significatives dans les dépenses selon le statut ou le niveau d'instruction des consommateurs ont été aussi observées (tableau VII). Les consommateurs salariés ont dépensé à chaque fois plus d'argent que les autres groupes $(p<0,05)$, et les consommateurs qui avaient un niveau d'instruction supérieur ont dépensé en moyenne plus d'argent que ceux de niveau inférieur et les analphabètes $(\mathrm{p}<0,01)$.

\section{Contraintes et innovations dans la filière}

\section{Contraintes}

Dans la production, les contraintes se situaient au niveau de la conduite des élevages. La faiblesse notoire des reproducteurs et les pertes importantes des porcelets avant sevrage ont été rapportées dans les entretiens. La pratique systématique du langueyage par les acheteurs pour détecter la présence de cysticerque suggérait une crainte réelle des opérateurs sur l'état d'infestation des animaux. Cette pratique systématique par les revendeurs et les collecteurs de porcs ne semblait pas connue des particuliers, ce qui contribuait à la dissémination de la cysticercose.

\section{Tableau VI}

Pratiques des consommateurs de viande porcine à Pala et Moundou au sud-ouest du Tchad

\begin{tabular}{|c|c|c|c|c|}
\hline \multicolumn{2}{|l|}{ Paramètre } & \multirow{2}{*}{$\begin{array}{c}\text { Ensemble } \\
98\end{array}$} & \multirow{2}{*}{$\begin{array}{c}\text { Moundou }(\mathrm{n}=37) \\
-\end{array}$} & \multirow{2}{*}{$\begin{array}{c}\text { Pala }(n=50) \\
-\end{array}$} \\
\hline Implantation des lieux de consommation (\%) & Autour des débits de boissons & & & \\
\hline & Eloigné & 2 & - & - \\
\hline \multirow[t]{2}{*}{ Type de débit de boisson (\%) } & Autour des cabarets & 78 & - & - \\
\hline & Autour des bars & 20 & - & - \\
\hline Fréquence consommation / semaine & & $4,36 \pm 2,65$ & $4,96 \pm 2,73^{\mathrm{a}}$ & $3,92 \pm 2,52^{b}$ \\
\hline \multirow[t]{5}{*}{ Produit consommé en $1^{\mathrm{er}}$ choix (\%) } & Viande grillée & - & 27 & 88 \\
\hline & Tête + oreille & - & - & 08 \\
\hline & Brochette & - & - & 04 \\
\hline & Viande frite & - & 51 & - \\
\hline & Viande au four & - & 22 & - \\
\hline \multirow[t]{3}{*}{ Produit consommé en $2^{e}$ choix $(n=23)(\%)$} & Soupe à la viande & 56,5 & - & - \\
\hline & Côtelette frite & 26,1 & - & - \\
\hline & Tête et oreille & 17,4 & - & - \\
\hline
\end{tabular}

a,b Les moyennes sur cette ligne suivies de lettres différentes sont significativement différentes au seuil de $5 \%$. 


\section{Tableau VII}

Dépenses moyennes (FCFA) par jour pour la consommation de viande porcine selon la catégorie de client et le niveau d'instruction à Pala et Moundou au sud-ouest du Tchad

\section{Dépense par catégorie de consommateur}

\section{Catégorie}

Moyenne

Salarié $(n=22)$

Producteur $(\mathrm{n}=18)$

Petit métier $(\mathrm{n}=23)$

Etudiant + élève $(n=24)$
$350,00 \pm 319,60^{\mathrm{a}}$

$186,11 \pm 85,42^{b}$

$221,74 \pm 99,80^{b}$

$247,92 \pm 127,24^{b}$
Dépense par niveau d'instruction

\begin{tabular}{ll} 
Niveau & \multicolumn{1}{c}{ Moyenne } \\
\hline Analphabète $(n=4)$ & $262,50 \pm 188,75^{\mathrm{b}}$ \\
Alphabétisé $(\mathrm{n}=4)$ & $225,00 \pm 50,00^{\mathrm{b}}$ \\
Primaire $(\mathrm{n}=28)$ & $182,14 \pm 72,28^{\mathrm{b}}$ \\
Secondaire $(\mathrm{n}=35)$ & $247,14 \pm 157,15^{\mathrm{b}}$ \\
Supérieur $(\mathrm{n}=16)$ & $400,00 \pm 325,06^{\mathrm{a}}$
\end{tabular}

${ }^{\mathrm{a}, \mathrm{b}}$ Les colonnes suivies de lettres différentes sont significativement différentes au seuil de $5 \%$.

Les pratiques de transformation et le cadre de consommation présentaient dans l'ensemble des conditions peu sanitaires. Bien que le prix des produits transformés ait été à la portée des consommateurs, la présentation des produits et l'emplacement le plus souvent côte à côte des lieux de transformation et de consommation restreignaient la fréquentation de ces derniers. Les consommateurs privilégiaient la qualité des produits proposés, accordant moins d'importance aux conditions sanitaires des lieux.

\section{Innovations}

Les principales innovations apparues dans la production de porc à Pala ont concerné la restructuration des porcheries suite à la formation des porciculteurs membres des groupements et aux meilleures pratiques d'élevage. En outre, une porcherie améliorée à titre d'exemple et un abattoir privé de taille moyenne financés par le Projet d'appui aux services agricoles et aux organisations de producteurs (PSAOP) ont été réalisés en 2005. De fait, plus de $50 \%$ des groupements ont été mis en place en 2004 et 2005 avec l'appui du PSAOP. Cette dynamique d'innovation se poursuit et vise à la fois à dynamiser la production et la commercialisation des porcs. A Moundou, l'innovation majeure dans la filière a été la constitution d'un groupement la Société d'élevage et de commercialisation des viandes et de leurs sous-produits (Secov) - de 30 éleveurs de porcs avec un effectif d'environ 300 porcs pour la production et la vente. Une boucherie-charcuterie moderne ouverte par un opérateur tchadien pour la transformation et la formation a été notée. Elle a été mise en place avec l'appui des prestataires de service de la base pétrolière de Komé (Doba). A la demande de cette base, cette boucherie-charcuterie a formé neufs jeunes aux techniques de la transformation de la viande porcine. A la fin des années 1990 et au début des années 2000, certains transformateurs (notamment à Moundou) ont adopté de nouvelles pratiques de friture de la viande porcine très peu connues auparavant.

\section{DISCUSSION}

L'étude a caractérisé les différents segments de la filière porcine dans les deux villes des savanes du Tchad. Elle a permis de connaître et de comprendre la production, la transformation et la consommation de viande porcine. Elle a montré une filière en plein développement au regard de l'engouement des acteurs, des données socioéconomiques, des années de création des élevages, et des innovations réalisées. Néanmoins, ces éléments résultant d'une enquête ponctuelle demanderaient à être validés par une étude longitudinale.

\section{Production}

L'examen des profils des acteurs a montré qu'ils étaient relativement jeunes. Leur âge et niveau scolaire étaient très proches de ceux mis en évidence chez les acteurs de la filière porcine de la zone de N'Djaména (Mopaté et al., 2008 ; 2007b). Ces éléments constituent des atouts pour d'éventuelles actions de développement de la filière porcine autour de ces deux villes. Concernant le genre dans la production, les résultats de la ville de Moundou ont été identiques à ceux de N'Djaména (Mopaté et al., 2007b). En revanche à Pala, les femmes ont été plus nombreuses. Cette observation a mis en évidence l'importance accordée à l'élevage porcin par les familles dans la région du Mayo-Kebbi en général et en particulier dans sa partie ouest. Cette région joue un rôle de premier plan dans la diffusion du porc en zone soudanienne du Tchad (Mopaté et al., 2006). D'ailleurs, la proportion des femmes dans la production porcine sur nos sites d'étude a été plus importante que celle rapportée dans d'autres contextes d'Afrique de l'Ouest ou du Centre. Dans la zone périurbaine de Cotonou, par exemple, les femmes sont très faiblement représentées $(4 \%)$ dans ce secteur (Youssao et al., 2008). L'implication des femmes dans la production de porcs tant en milieu urbain que rural montre la dynamique d'une production à même de pallier leurs difficultés. L'élevage porcin apparaît ainsi comme une activité très importante pour les familles. La propriété des élevages a été surtout individuelle sur les deux sites de l'étude, comme c'est le cas $(84 \%)$ à Cotonou. L'échange des porcelets contre la livraison de drêches artisanales a contribué à la création de nouveaux élevages porcins dans des zones où de telles pratiques existent. Les mêmes observations sont faites dans la zone de N'Djaména (Mopaté, 2008). Les acteurs dans la transformation dans les deux villes avaient globalement le même âge moyen (31 ans) que celui observé dans la zone urbaine de Bobo-Dioulasso au Burkina Faso (Kiendrébeogo et al., 2012).

Les effectifs moyens des troupeaux ont été moins importants que ceux observés en Casamance au Sénégal (Ossebi et al., 2019). Il en était de même pour la taille de la portée à la mise bas. La proportion des truies (3 à 4) et des verrats (1) dans les troupeaux a été proche de celle observée à Cotonou (Youssao et al., 2008) mais inférieure à celle de cinq reproductrices rapportée en Casamance (Ossebi et al., 2019). La productivité numérique annuelle au sevrage de 11 têtes a été identique aux résultats obtenus à Garoua (Cameroun), supérieure à 8 porcelets notés à Bangui (Centrafrique), inférieure à 12 têtes observées antérieurement à Pala (Mopaté et al., 2010) et à 12-14 porcelets rapportés dans le bassin arachidier sénégalais (Buldgen et al., 1994). En revanche, le nombre moyen annuel de mises bas par truie a été semblable à ceux de 2 et 1,9 observés respectivement à Bangui et à Pala dans une étude antérieure (Mopaté et al., 2010) et en Casamance (Ossebi et al., 2019). Les meilleures performances de reproduction à Pala s'expliquaient sans doute par des pratiques d'alimentation relativement bonnes. Ces différences se sont retrouvées aussi dans la moyenne des pertes au sevrage qui a été plus élevée à Moundou. Cela s'est répercuté sur la productivité numérique qui a été meilleure à Pala. 


\section{Transformation}

Les produits transformés ont été dans l'ensemble les mêmes que ceux identifiés dans la zone de N'Djaména (viande grillée, viande frite, brochettes et soupe à la viande) (Mopaté et al., 2008), et dans d'autres villes comme Abidjan en Côte d'Ivoire, Ougadougou au Burkina Faso et Yaoundé au Cameroun (Mopaté et Kaboré-Zoungrana, 2013). Les particularités notées au niveau des deux villes ont concerné les côtelettes, la tête et les oreilles. C'était probablement pour valoriser au mieux la carcasse. Les lieux de consommation étaient aussi pour la plupart des débits de boisson, notamment des cabarets pour les boissons traditionnelles et des bars pour les autres. Les mêmes observations sont rapportées par Mopaté et al. (2008), Mopaté et Matna (2012), et Kiendrébeogo et al. (2012), respectivement à N'Djaména, Moundou et Bobo-Dioulasso. Cela indique des pratiques semblables des acteurs de ce segment de la filière, destinées à exploiter les possibilités qu'offrent ces débits de boisson dans la commercialisation des différents produits porcins transformés. En effet, les lieux de transformation sont le plus souvent intégrés aux lieux de consommation.

\section{Consommation}

Les pratiques de consommation hors foyer de la viande porcine sont récentes (moins de trente ans) et ont été introduites surtout du Cameroun par certains jeunes qui y ont séjourné. Elles se sont développées avec l'accroissement de la demande. Cette viande était auparavant consommée à la maison lors de festivités, de réception d'un hôte ou de travaux en groupe (Mopaté et Koussou, 2003). Les pratiques identifiées dans les deux villes ont été dans l'ensemble les mêmes que celles mises en évidence dans les points de grillades et les rôtisseries de N'Djaména, d'Abidjan, de Ougadougou, de Yaoundé et de Bobo-Dioulasso (Mopaté et al., 2008 ; Mopaté et Kaboré-Zoungrana, 2013 ; Robineau, 2018). On comprend également les stratégies d'adaptation des transformateurs à la demande à travers la diversité des produits transformés, comme les côtelettes, la tête, les oreilles et les brochettes, en plus des viandes frites ou grillées, et de la soupe à la viande. Les fonctionnaires, les étudiants et les personnes exerçants des petits métiers étaient largement majoritaires dans les différents lieux de restauration, contrairement aux élèves et aux agriculteurs. Le nombre de personnes dans les groupes de consommateurs fréquentant de manière collective ces lieux dans les deux villes (trois personnes) était sensiblement le même qu'à N'Djaména (Mopaté et al., 2008). Les dépenses moyennes pour la consommation des produits porcins transformés étaient cependant faibles.

\section{Innovations}

Les innovations ont été introduites à la faveur de deux événements : l'ouverture des marchés camerounais aux porcs du Tchad à la fin des années 1990 et au début des années 2000 (Koussou et Duteurtre, 2002), et l'exploitation pétrolière dans le bassin de Doba dans les années 2000. La production à Pala était surtout orientée vers les marchés camerounais, et celle de Moundou vers l'approvisionnement de la base pétrolière de la ville de Komé, suite à la hausse de la demande de cette dernière. Face à ces opportunités, les acteurs se sont organisés pour satisfaire la demande, soit de leur propre initiative soit avec l'appui des projets de développement. La hausse de la demande en lien avec l'urbanisation a créé des opportunités, et impose un changement et une organisation de la production et de la transformation (Lossouarn, 2003).

\section{CONCLUSION}

Leétude a permis de caractériser la filière porcine à Pala et à Moundou au sud-ouest du Tchad. Comme bien d'autres filières agroalimentaires en Afrique au sud du Sahara, la filière porcine dans ces deux centres urbains contribue à la sécurité alimentaire, à l'amélioration de la nutrition à travers la consommation de protéines animales, à la lutte contre la pauvreté et à la diversification des activités génératrices de revenus. Une demande croissante en viande porcine entraîne des innovations dans la production et la transformation pour y faire face. La bonne prolificité reconnue aux porcs et la marge d'amélioration de sa production en font un animal de choix. La viande porcine est consommée par toutes les couches sociales pour lesquelles il n'y a pas d'interdit religieux. Les transformateurs développent des stratégies pour écouler rapidement leurs produits. La diversité des préparations et surtout les prix fixés les rendent accessibles à toutes les bourses. Pour la qualité de la production, une étude de détermination de l'impact des infestations de cysticerques sur la production doit être entreprise. Il en est de même des pratiques à risque de la conduite des élevages, de la transformation et de la consommation de cette viande. La transformation de la viande porcine génère des emplois, résorbant ainsi le chômage. Elle favorise également la vente des boissons à travers l'activité commerciale des cabarets et des bars. Les paramètres de production relativement faibles orientent les perspectives de recherche vers l'amélioration de la conduite de cet élevage. Une organisation socioprofessionnelle conséquente de cette activité est nécessaire pour une meilleure dynamique de la filière et une amélioration des revenus des acteurs.

\section{Déclaration des contributions des auteurs}

YML : conception et planification, analyse des données et rédaction de la première version ; MT et ABB : participation à l'élaboration du questionnaire et à la révision du manuscrit ; $\mathrm{AN}$ : participation à la planification de l'enquête de terrain et au recueil des données.

\section{REFERENCES}

Bricas N., Seck P.A., 2004. L'alimentation des villes du Sud : les raisons de craindre et d'espérer. Cah. Agric., 13 (1) : 10-14

Buldgen A., Piraux M., Dieng A., Schmit G., Compère R., 1994. Les élevages de porcs traditionnels du bassin arachidier sénégalais. Rev. Mond. Zootech., 80/81 (3-4) : 63-70

Cirad-Emvt, 1999. Socio-économie de l'élevage ovin périurbain (Sécoville). Cirad-Emvt, Montpellier, France,102 p. (Rapport ; 99-020)

Diao M.B., 2004. Situation et contraintes des systèmes urbains et périurbains de production horticole et animale dans la région de Dakar. Cah. Agric., 13 (1) : 39-49

Dongmo T., Gockowski J., Hernandez S., Awono L.D.K., Mbang à Moudon R., 2005. L'agriculture périurbaine à Yaoundé : ses rapports avec la réduction de la pauvreté, le développement économique, la conservation de la biodiversité et de I'environnement. Tropicultura, 23 (3) : 130-135, doi : 10.7202/602155ar

Fall A., Ba Diao M., Bastianelli D., Naniogo A. 2004. La gestion concertée et durable des filières animales urbaines. In : Développement durable de I'agriculture urbaine en Afrique francophone. Enjeux, concepts et méthode (éds Olanrewaju B., Moustier P., Mougeot L.J.A., Fall A.). Cirad, Montpellier, France, 96-122

Guerin H., Faye B., 1999. Spécificité de la problématique périurbaine pour les systèmes d'élevage. In : Actes atelier Agriculture périurbaine en Afrique subsaharienne (éds Moustier P., Mbaye A., De Bon H., Guerin H., Pages J.), Cirad-Coraf, Montpellier, France, 20-24 avr. 1998, 43-49

Kiendrébeogo T., Mopaté Logténé Y., Kondombo S.R., Kaboré-Zoungrana C.Y., 2012. Approvisionnement en porcs vifs et viande porcine de la ville de Bobo-Dioulasso (Burkina Faso). J. Agric. Environ. Int. Dev., 106 (2) : 105-122

Koussou M.O., Duteurtre G., 2002. Les facteurs de compétitivité de la filière porcine dans le bassin du Logone. In : Colloq. Systèmes agro-alimentaires localisés, Syal, Cirad, Montpellier, France, oct. 2002

Lossouarn J., 2003. Stratégies dans les filières animales. Prod. Anim., 16 (5) : 317-324

Mfoukou-Ntsakala A., Michel Bitémo M., Speybroeck N., Van Huylenbroeck G., Eric Thys E., 2006. Agriculture urbaine et subsistance des ménages dans une zone de post-conflit en Afrique centrale. Biotechnol. Agron. Soc. Environ., 10 (3) : 237-249 
Mopaté L.Y., 2008. Dynamique des élevages porcins et amélioration de la production en zones urbaine et périurbaine de la ville de N'Djaména (Tchad). Thèse Doct., Prod. Anim., Université polytechnique, BoboDioulasso, Burkina Faso, 245 p.

Mopaté L.Y., Kaboré-Zoungrana C.Y., 2013. Production, commerce et consommation de la viande porcine dans quelques villes d'Afrique de I'Ouest et du Centre. Rev. Sci. Tchad, 13 (2) : 34-44

Mopaté L.Y., Kaboré-Zoungrana C.Y., Gongnet P.G., 2007b. Dynamique des élevages porcins urbains : accroissement, caractéristiques des producteurs et des effectifs à N'Djaména (Tchad). Ann. Univ. N'Djaména (Tchad), Sér. C, Sci. Exactes Appl. Santé, 2 : 75-91

Mopaté L.Y., Koussou M.O., 2003. L'élevage porcin, un élevage ignoré mais pourtant bien implanté dans les agrosystèmes ruraux et périurbains du Tchad. In : Actes Colloq. Savanes africaines : des espaces en mutations, des acteurs face à de nouveaux défis (éds Jamin J.Y., Seyni Boukar L., Floret C.), Garoua, Cameroun, 27-31 mai 2002, 9 p.

Mopaté L.Y., Koussou M.O., Kaboré-Zoungrana C.Y., 2006. L'élevage porcin au Tchad : bilan de l'introduction, de l'amélioration et de la diffusion des races exotiques. Bull. Info. Ress. Génét. Anim., 38 : 37-48, doi : 10.1017/ S101423390000208X

Mopaté L.Y., Koussou M.O., Kaboré-Zoungrana C.Y., 2007a. Dynamique de la production porcine à N'Djaména (Tchad) : évolution des abattages, des poids carcasses durant cinq décennies passées et prévisions actuelles. Rev. Sci. Tchad, 9 (2) : 60-70

Mopaté L.Y., Koussou M.O., Kaboré-Zoungrana C.Y., 2008. Consommateurs et consommation de la viande porcine en hors-foyer à N'Djaména (Tchad). In Gouvernance et approvisionnement des villes, tome I (éds Parrot L., et al.). L'Harmattan, Paris, France, 199-208

Mopaté L.Y., Koussou M.O., Nguertoum A.E., Ngo Tama A.C., Lakoueténé T., Awa N.D., Mal Mal H.E., 2010. Caractéristiques et performances des élevages porcins urbains et périurbains des savanes d'Afrique centrale : cas des villes de Garoua, Pala et Bangui. In : Actes Colloq. Savanes africaines en développement : innover pour durer (éds Seinyboukar L., Boumard P.), Garoua, Cameroun, 20-23 avril 2009. Prasac, N’Djaména, Tchad / Cirad, Montpellier, France, 9 p.

\section{Summary}

Mopaté Logténé Y., Tellah M., Bechir A.B., Nayo A. Characterization of the swine sector in the cities of Pala and Moundou in the savanna zone of Southwestern Chad

The study characterized the different segments of the swine sector in the cities of Pala and Moundou in the savanna zone of Chad. Cross-sectional and retrospective surveys, combined with direct observations, were applied to producers, processors and consumers in these cities. A total of 1318 pigs were counted, including 354 females of reproductive age. The average number of parturitions per year was 1.8 and the litter size 7.2 piglets. The annual numerical productivity per female was 11 piglets. Concerning the carcasses processed annually, more than half were processed in Moundou, the second-largest city in Chad. In Pala, consumers preferred grilled pork. In Moundou, preferences were divided between fried pork and baked or grilled pork. The results also showed that pig farming was aimed at improving family income through the sale of animals, and the production of self-consumed animals. Through the diversity of processed products, food processing and preparation activities, the actors in the sector provided the consumers with pork products at a relatively moderate cost. The major innovations observed in the sector were an improved pigsty, a private slaughterhouse, a breeding and pork marketing company, and a butchery's shop and delicatessen for processing and distribution. Production, processing and marketing of pork have generated employment and improved the income of the different actors in the sector.

Keywords: swine, food supply chain, animal production, consumer behaviour, Chad
Mopaté L.Y., Matna M.M., 2012. Approvisionnement, transformation et consommation hors-foyer de porcs dans la ville de Moundou (Tchad). Rev. Sci. Tchad, 11 (spécial) : 53-62

Moustier P., Moumbélé M., Huat J., 2004. La gestion concertée et durable des filières maraîchères urbaines. In : Développement durable de l'agriculture urbaine en Afrique francophone. Enjeux, concepts et méthode (éds Olanrewaju B., Moustier P., Mougeot L.J.A., Fall A.). Cirad, Montpellier, France, 66-95

Ossebi W., Ayssiwede S.B., Nimbona F., Malou R., Djetiin A.E., Diop M., Missohou A., 2018. Obstacles to the development of the pig value chain in Casamance (Senegal): what do actors of the subsector say? Rev. Elev. Med. Vet. Pays Trop., 71 (1-2): 15-22, doi: 10.19182/remvt.31286

Ossebi W., Ayssiwede S.B., Nimbona F., Malou R., Djettin A.E., Diop M., Missohou A., 2019. Zootechnical and economic analysis of pig farming systems in Casamance (Senegal). Rev. Elev. Med. Vet. Pays Trop., 72 (1): 1322, doi: 10.19182/remvt.31258

Robineau O., 2018. Rearing pigs in a West African city: Arrangements between actors to manage the proximity between the city and livestock farming. Rev. Elev. Med. Vet. Pays Trop., 71 (1-2): 23-31, doi: 10.19182/remvt.31288

SPSS, 2009. Statistical package for social sciences, Vers. 17.0. SPSS, Chicago, IL, USA

Temple L., Moustier P., 2004. Les fonctions et contraintes de l'agriculture périurbaine de quelques villes africaines (Yaoundé, Cotonou, Dakar). Cah. Agric.; 13 (1) : 15-22

Touraille C., Monin G., 1984. Comparaison des qualités organoleptiques de la viande de porcs de trois races : large White, Landrace français, Landrace belge. In : 16 ${ }^{\text {es }}$ Journées Recherche porcine, INERA-ITP, 1-3 fév. 1984, 75-80

Touré G., Ouattara Z., 2001. Elevage urbain des ovins par les femmes à Bouaké, Côte d'Ivoire. Cah. Agric., 10 (1) : 45-49

Youssao A.K.I., Koutinhouin G.B., Kpodekon T.M., Bonou A.G., Adjakpa A., Dotcho C.D.G., Atodjinou F.T.R., 2008. Pig production and indigenous genetic resources in suburban areas of Cotonou and Abomey-Calavi in Benin. Rev. Elev. Med. Vet. Pays Trop., 61 (3-4): 235-243, doi: 10.19182/ remvt.9995

\section{Resumen}

Mopaté Logténé Y., Tellah M., Bechir A.B., Nayo A. Caracterización del sector porcino en las ciudades de Pala y Moundou en la zona de las sabanas del sud oeste de Chad

El estudio caracterizó los diferentes segmentos del sector porcino de las ciudades de Pala y Moundou en la zona de las sabanas de Chad. Se llevaron a cabo encuestas transversales y retrospectivas, junto con observaciones directas, a productores, procesadores y consumidores distribuidos en esas dos ciudades. En total, se contaron 1318 cerdos, de los cuáles 354 hembras en edad de reproducción. El número de partos promedio por año fue de 1,8 y el tamaño de la camada de 7,2 lechones. La productividad numérica anual por hembra fue de 11 lechones. Del total de carcasas procesadas anualmente, más de la mitad lo fue en Moundou, segunda ciudad de Chad. En Pala, los consumidores prefirieron la carne a la parriIla. En Moundou, las preferencias fueron compartidas entre la carne frita y la carne al horno o a la parrilla. Los resultados muestran también que el objetivo de la cría de cerdos era el de mejorar los ingresos de la familia gracias a la venta de animales y a la producción de animales para consumo propio. Mediante la diversidad de productos procesados, las actividades de transformación y de preparación alimenticias, los actores del sector pusieron a la disposición de los consumidores, alimentos cárnicos a un costo relativamente moderado. Las innovaciones mayores observadas en el sector fueron una porqueriza mejorada, un matadero privado, una sociedad de cría y de comercialización de carnes y una carnicería-charcutería para la transformación y la distribución. La producción, transformación y comercialización de la carne porcina generaron empleos y mejoraron los ingresos de los diferentes actores del sector.

Palabras clave: cerdo, cadena de suministro alimentario, producción animal, comportamiento del consumidor, Chad 